MATEC Web of Conferences 22,02021 (2015)

DOI: $10.1051 /$ matec conf/ 20152202021

(C) Owned by the authors, published by EDP Sciences, 2015

\title{
Research Overview on Wireless Power Transmission Technology
}

\author{
Tao Li, Liheng Wu \& Zheng Chen \\ Institute of Crustal Dynamics, CEA, Beijing, China
}

\begin{abstract}
According to the latest researches, this paper outlines the development of wireless power transmission and introduces the latest applications of wireless power transmission in life. To describe the wireless power transmission technologies in detail, the paper presents the short-range, medium-range and remote wireless power transmission, respectively. In addition, the paper also depicts some unique properties of wireless power transmission system to make readers understand WPT system better. At last, the existing problems and developing trends are presented.
\end{abstract}

Keywords: Wireless power transmission, Electromagnetic induction, Electromagnetic resonance, Short-range, Medium-range, Remote.

\section{INTRODUCTION}

Wireless power transmission is a technology that utilizes radio to transmit power energy, mainly through electromagnetic induction, electromagnetic resonance, $\mathrm{RF}$, microwave, laser to realize non-contact power transmission. With the development of power electronic devices, power conversion and control technology, the wireless power transmission technology has achieved a gradual breakthrough in the conversion rate, low radiation and other aspects. A wireless power transmission system is defined as one that efficiently transmits electric power from one point to another through the vacuum of space or the Earth's atmosphere without the use of wires or any other substance. Wireless power transmission is also often referred to as "beamed power transmission". Wireless power transmission is different from free-space point to point communication systems by the comparatively high levels of power being handled, by its high efficiency, and by its technology and physical components that are distinctly different from those used in communication systems.

The wireless power transmission shows a good prospect in the military, communications, industrial, medical, transportation, electric power, aerospace, energy environmental protection and other areas. This technology has rapidly become the hot pursuing topic for research institutions in recent years, and then has made some major breakthroughs in electric vehicles, body implantable medical devices, small robots, portable mobile device chargers. Wireless power transmission includes power transfer at microwave frequencies as well as at optical frequencies. Wireless power transmission at microwave frequencies preceded any development at optical frequencies and at the present time the efficiency at microwave frequencies is much greater than at laser frequencies and the system power handling capability is much greater. In recent years, the global wireless power transmission market size has increased, according to HIS iSuppli data, the market revenue of wireless charging equipment reached \$ 120 million in 2010, and it will reach \$ 23.7 billion in 2015. Starting in 2011, the global sales of wireless charging module has made rapid growth, and will grow to 923 million in 2019 .

Because of the different power distance that wireless power transmission can achieve, wireless power transmission can be broadly divided into short-range wireless power transmission, medium-range wireless power transmission and remote wireless power transmission. Short-range wireless power transmission uses magnetic coupling of electromagnetic induction for short-transmission. Medium-range power transmission uses the energy of electromagnetic waves RF or non-radiative resonant magnetic coupling and other forms for medium-range transmission. Remote power transmission uses microwave or laser energy to form long-distance transmission-transmitted to the distal end of the receiving antenna, and then conducts rectifier and modulation for further uses. So, this paper will introduce the three wireless power transmission technologies, respectively.

\section{SHORT-RANGE WIRELESS POWER TRANS- MISSION}

Short-range wireless power transmission can be achieved by electromagnetic induction. This approach requires the magnetic field as a transmission medium, with the use of magnetic coupling between the transmitting coils and receiving coils to transmit energy. Using a transformer coupling, the primary and secondary coils can generate induced currents which may form an alternating electric field in the medium, so that power can be transmitted via the most non-metallic materials and the energy can be trans- 
ferred from the transmitter to the receiver, enabling wireless transmission of electricity. The equivalent circuit of WPT system can be seen in Figure 1. The advantage of this transmission is simple structure, relatively low cost, safe and reliable technology. The disadvantage is that the power transmission distance is small and subject to a maximum of about $10 \mathrm{~cm}$, and can generally be used in electronic devices of power small. Currently common applications include inductive charging of electric toothbrush, mp3, universal wireless power pad and other small power electronic devices.
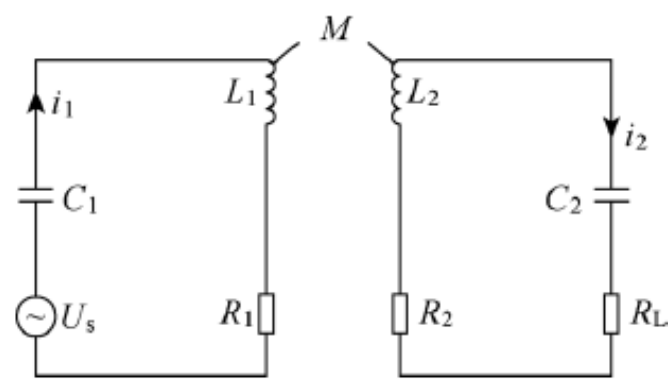

Figure 1. Equivalent circuit of WPT system

Take the universal wireless power pad for example and we will introduce it in detail. In 2003, the Splash Power Company in England Cambridge invented the wireless recharging system, which is also using electromagnetic induction to transmit power, and the receiver Splash Module can be deployed in the charging end - mobile phone or laptop, and the power transmitter is configured to charger. Early in 2005, this commercial wireless charger with the size of a mouse pad was put in market. As long as the portable terminal mounts power receiver, it can be charged. (See Figure 2)

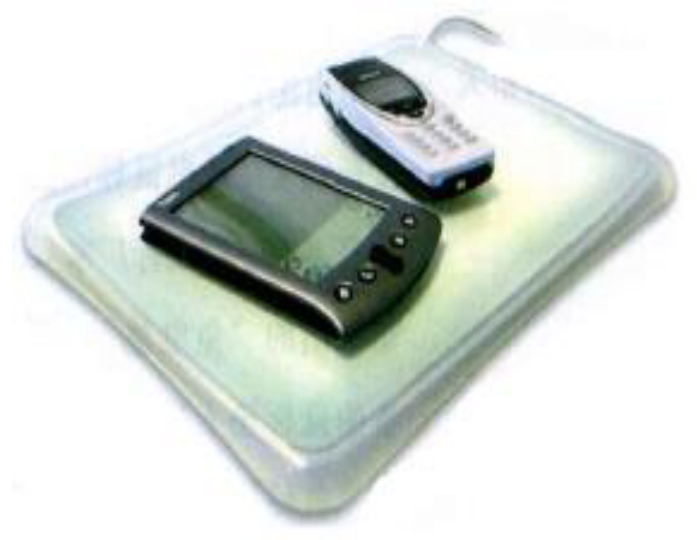

Figure 2. Universal wireless power pad
Now the widely used transformer is working based on the principle of electromagnetic induction, which consists of a core and two coils (the primary coil and the secondary coil). When an alternating voltage is applied across the primary coil, the core will generate an AC magnetic field, whereby the secondary coil will generate an $\mathrm{AC}$ voltage in a same frequency, and then power is transmitted from the input circuit to the output circuit. Taking the commercially available non-contact charging system for example, the power supply coil which is connected to the power and the receiver coil which is in electronic products are in two separate devices, and the power is transmitted through the induction coil, which is similar to an transformer which is not tightly close in inter-coils.

\section{MEDIUM-RANGE WIRELESS POWER TRANSMISSION}

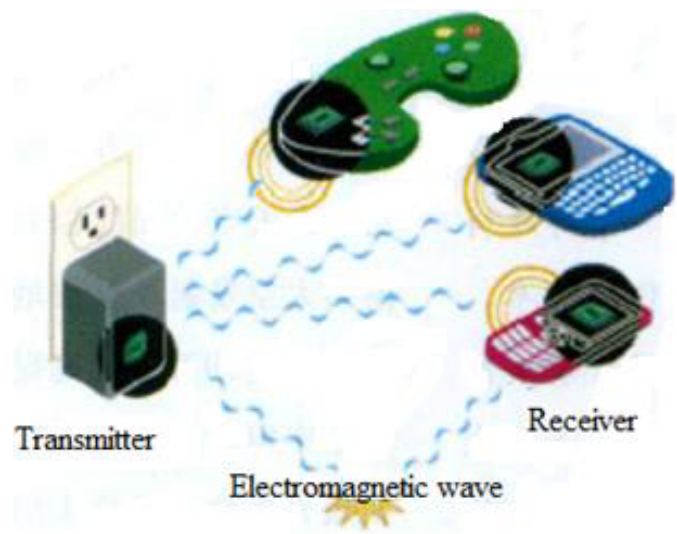

Figure 3. Schematic diagram of a wireless power supply system

As is known to all, the electromagnetic waves whose frequency range is between $75 \mathrm{kHz}$ and $10 \mathrm{GHz}$ is called radio waves, and it can be used to transmit radio and television programs, and conduct fax and communications, but its ability to transmit electrical energy is unfamiliar. Usually, electromagnetic waves transmission in free space during the process of energy transferring will emit in all directions, difficult to concentrate and has poor orientation, and thus the efficiency of the power supply is a problem. In addition, there is also a concern that it will cause space electromagnetic pollution. Some people think that electromagnetic waves can wirelessly transmit a longer distance, but transportation energy is limited, and the transmission power is relatively low. Related research of Powercast uses antenna technology with small loss of electromagnetic wave, by means of the diode, the non-contact IC card and the wireless electronic labels, to achieve a higher efficiency of wireless power transmission. Powercast said that they started the work in 2003 , and the technology was approved by FCC. 
The whole system consists of two modules, one is "Power Caster" transmitter which can be plugged into the power outlet, and the other is "Powerharvester" receiver which can be embedded in electronic products. (Seen in Figure 3)

Medium-range wireless power transmission utilizes electromagnetic resonance coupling principle or radiofrequency electromagnetic to achieve. The transmission distance of this transmission type can run up to 8 times of that in the induction coil within a radius, and it uses the receiving antenna and launch site with the same electromagnetic frequency as its natural frequency to cause electromagnetic resonance, in order to generate strong electromagnetic coupling to achieve the medium distance electricity transmission. This transmission distance is longer, with its power up to kilowatts. In the International Consumer Electronics Show in 2010, the Haier exhibited the use of wireless power technology for high-definition television. Its "television of no end" used electric magnetic coupling technology, and this TV has a built-coil chassis so that it can receive a $100 \mathrm{~W}$ power supply at a distance of $1 \mathrm{~m}$. The distance depending on the power supply with the size of the coil can be powered to farthest 3 to 5 times the diameter of the coil distance. This technology can provide wireless power transmission for mobile phones, laptops and hearing aids.

\section{REMOTE WIRELESS POWER TRANSMIS- SION}

The remote wireless power transmission uses microwaves, lasers and other forms of energy to achieve the long-distance propagation of power. The interference immunity ability of microwaves and laser is strong, through effective adjustment, rectifier and other manners, it can be transmitted to the distal end of the receiving device in order to achieve long-distance power transmission. The infrastructure of this way is expensive, and it can be used in long-distance, large-scale power transmission, so most are used in space technology areas, such as the energy transfer of man-made satellites, space stations and between spacecraft.

Theoretically, the shorter radio wave length is, the better its orientation is, the smaller the dispersion is, so it can be used in the form of microwaves or laser to achieve the long-distance transmission of electrical energy. Then, it can make great significance for the development and utilization of new energy to solve future problems of energy shortages.

Therefore, many countries have continued to make research in this area. In 1968, the American engineer Peter Glaser proposed the concept of space solar power, and its vision is to build a solar power base in outer space by microwave energy back to Earth. In 1979, the NASA and the US Department of Energy's solar plan jointly proposed the establishment of SPS solar system satellite base. SPS (Solar Power satellite) is a solar power satellite in geostationary orbit, where the
Sun's energy is about 1.4 times that on Earth. It is predicted that an SPS solar cells can load DC output power of $10 \mathrm{GW}$, battery output power via microwave oscillator to convert electricity from power transmission antenna to the Earth's surface by microwave $(2.45 \mathrm{GHz})$ in the form of wireless transmission. When a receive antenna is placed on Earth, which is composed of a half wavelength dipole antenna, a rectifier diode, a low pass filter and bypass capacitors, electric power can be received up to about 5GW. Currently, the SPS construction method, the radiation characteristics of the antenna, the attitude control of microwave transmitting devices, microwave propagation characteristics of space, and the safe security systems in order to ensure failure are all technical problems to be solved in the future.

\section{UNIQUE PROPERTIES OF WIRELESS POWER TRANSMISSION SYSTEM}

A wireless power transmission system often has many unique properties that almost guarantee it as an important element, and probably a crucial one in the development of space. According to a number of researchers' views, we can find that these unique properties usually include the following ones:

a) Energy can be transferred at the velocity of light.

b) The direction of energy transfer can be changed rapidly.

c) The mass of the power converters at the system terminals can be low because of operation at microwave frequencies.

d) No mass, either in the form of wires or ferrying vehicles, is required between the source of energy and the point of consumption.

e) No energy is lost in its transfer through the vacuum of space, and little is lost in the Earth's atmosphere at the longer microwave wavelengths.

f) Energy transfer between points is independent of a difference in gravitational potential between those points.

As is known to all, wireless power transmission technology can apply in many electricity devices, and it can transfer power and energy at the velocity of light without the use of wires or any other substance. The vacuum of space may be regarded as a superconducting medium. For there are no losses when the microwave energy propagates through space, energy can be transferred over long distances without loss, such as from geosynchronous orbit to Earth, thus making some new apparatus possible like the solar power satellite. In addition, to transfer electric energy from Earth to space to be used in high specific impulse electric thrusters for journey from low-Earth orbit to high-Earth orbit or even geosynchronous orbit and then back, the property that energy transfer between points is independent of a difference in gravitational potential between those points makes it possible. This ability can reduce by a factor of twenty the mass of chemical propellant that 
would otherwise be used and would have to be brought up from the Earth to low-Earth orbit through the gravitational barrier at high expense.

\section{THE EXISTING PROBLEMS AND DEVELOP- ING TRENDS}

Wireless power transmission technology is not a new concept, but the introduction of new technologies and new applications has gradually made it become a new research discipline. Especially, wireless power transmission technology has unique advantages that a traditional supply line technology cannot provide. The development and further breakthrough of this technology will have a profound impact on electric vehicles, medical, industrial, electronics and other fields. In recent years, although wireless power transmission technology based on resonant magnetic coupling has gradually been on the rise, in fact, there are still many problems to be solved. For example, the theory is not perfect, although the existing theory can provide some theoretical support for actual research, and the research on the system characteristics, parameter optimization, and spatial distribution of the electromagnetic still has no more stringent analysis methods.

High-frequency power supply and rectifier technology has no good solution yet, and the existing high-frequency power solution still has defects such as widespread inefficiency, design complexity and others. The control and optimization problems of system should be solved, and the problem of instability high quality factor resonator coil brings to the system also has not been effectively addressed. Of course, the way that can currently achieve medium-distance wireless energy transfer still has great potential for development.

\section{CONCLUSION}

As the wireless power transmission technology continues to evolve and mature, our future life can expect to get rid of the devices power cord bound of mobile phones, cameras, laptops and other mobiles to enjoy the wireless power at the airport, railway stations, hotel offers and a variety of places. This paper describes the development of wireless power transmission and the latest applications of wireless power transmission in life. Especially, the paper presents the short-range, medium-range and remote wireless power transmission in detail, respectively. Then, this paper also concludes some unique properties that almost guarantee it as an important element, and probably a crucial one in the development of space. At last, the existing problems and developing trends are presented. In the long term, the technology has a wide range of potential applications.

\section{ACKNOWLEDGEMENTS}

This paper was supported by the Special Scientific Research Found of Earthquake (Grant No.201408006) and the Institute of Crustal Dynamics, CEA, Basic scientific Special Fund (ZDJ2013-06).

\section{REFERENCES}

[1] Zeng X. 2010. Study on the technology of wireless power transmission. Silicon Valley, (10): 82-82.

[2] Liu Y. 2008. Wireless power transmission technology: the creation myth of the future space. China Electronic Business: Basic Electronics, 8(11): 70-75.

[3] Yang Q, Chen H. \& Xu G. 2010. Research progress of non-contact power transmission technology. Transactions of China Electro technical Society, 25 (7): 6-13.

[4] Liu C, et al. 2014. Robust $\mathrm{H} \infty$ control for satellite attitude control system with uncertainties and additive perturbation. International Journal of Science, 1(2): 1-9.

[5] Shi K. \& Sun Z, et al. 2015. Design of microsatellite attitude control with multiplicative perturbation of controller, Control and Decision Conference (2015 CCDC), The 27th Chinese. IEEE.

[6] Chen Q. 2014. Abroad progress of wireless power transmission technology. Shanghai Information Technology, (1).

[7] Zhu H, Xu C. \& Dou C. 2014. Discussion on the wireless power transmission and new applications. Information Systems Engineering, (3): 94-94.

[8] Liu C. \& Wang F. 2014. In-orbit estimation of inertia parameters of target satellite after capturing the tracking satellite. Intelligent Control and Automation (WCICA), 2014 11th World Congress on. IEEE, pp: 3942-3947.

[9] McSpadden J O. \& Mankins J C. 2002. Space solar power programs and microwave wireless power transmission technology. Microwave Magazine, IEEE, 3(4): 46-57.

[10]Brown W C. 1996. The history of wireless power transmission. Solar Energy, 56(1): 3-21.

[11]Huang H, Huang X. \& Tan L, et al. 2011. Research on transmitter and receiver of wireless power transmission based on magnetic resonance coupling. Advanced Technology of Electrical Engineering and Energy, 1: 008.

[12] Matsumoto H. 2002. Research on solar power satellites and microwave power transmission in Japan. Microwave Magazine, IEEE, 3(4): 36-45.

[13]Brown W C. 1996. The history of wireless power transmission. Solar Energy, 56(1): 3-21.

[14]Liu C. \& Shi K, et al. 2014. Mass and mass center identification of target satellite after rendezvous and docking, Intelligent Control and Automation (WCICA), 2014 11th World Congress on. IEEE, pp: 5802-5807.

[15] Tan L, Huang X. \& Huang H, et al. 2011. Transfer efficiency optimal control of magnetic resonance coupled system of wireless power transfer based on frequency control. Science China Technological Sciences, 54(6): 1428-1434. 